\title{
09
}

\section{Самоиндуцированная прозрачность в монослое черного фосфора}

\author{
(c) Г.Т. Адамашвили
}

Грузинский технический университет, Тбилиси, Грузия

E-mail: guram_adamashvili@ymail.com.

Поступило в Редакцию 13 сентября 2017 г.

Построена теория оптического солитона самоиндуцированной прозрачности в монослое черного фосфора (фосфорена). Получены явные аналитические выражения для поверхностного солитона в фосфорене и других анизотропных двумерных материалах. Показано, что анизотропная проводимость фосфорена приводит к экспоненциальному затуханию амплитуды солитона поверхностной волны, которая сильно зависит от направления распространения импульса. Максимальное затухание амплитуды получено вдоль направления „кресло“.

DOI: 10.21883/PJTF.2018.08.45970.17037

Фосфорен - двумерный атомный слой черного фосфора (ЧФ) — вызвал значительный интерес благодаря его замечательным оптическим характеристикам. Недавним достижением является экспериментальная реализация монослоя ЧФ, которая стимулировала интенсивные теоретические и экспериментальные исследования (см., например, [1,2]). В отличие от графена [3] в фосфорене атомы расположены так, что образуют сморщенную конфигурацию, которая приводит к сильным анизотропным оптическим свойствам в плоскости. Эксперимент доказал сильно анизотропный характер проводимости фосфорена [4]. Анизотропные оптические свойства ЧФ и других фосфороподобных материалов $[5,6]$ делают их важными объектами для исследований и приложений. Двумерные анизотропные материалы вызвали значительный интерес из-за их применения в плазмонике, где можно возбуждать поверхностные плазмон-поляритоны (ППП). Одним из важных свойств ППП является их способность концентрировать энергию волны в области границы раздела, обеспечивая сильную связь ППП с фосфореном. По сравнению с графеновым плазмоником в фосфорене свойства ППП более разнообразны и интересны, поскольку дополнительно демонстрируют сильные анизотропные свойства. Одной из горячих 
тем исследования является формирование резонансных солитонов или солитоноподобных нелинейных ППП. Нелинейные волны в двумерных структурах интенсивно изучались как теоретически [7-11], так и экспериментально [12-16]. Резонансные солитоны и бризеры ППП могут формироваться с помощью явления самоиндуцированной прозрачности (СИП) в поглощающей среде, а в усилителе может распространяться солитоноподобный импульс (л-импульс) [17]. Теоретические исследования СИП для солитонов были проведены в изотропном монослое графена для ППП [7] и волноводных мод [8], а также для бризера ППП [9]. В этих работах показано, что проводимость графена приводит к экспоненциальному затуханию интенсивностей солитонов и бризеров в процессе распространения.

В последнее время скалярные и векторные солитоны, а также некоторые солитоноподобные импульсы были экспериментально исследованы в тонких пленках ЧФ и поглотителе на основе квантовых точек ЧФ в легированном эрбием волокне лазерной полости [12-16]. Эти эксперименты показали, что параметры солитонов сильно зависят от направления распространения волны, что является следствием анизотропии ЧФ. Солитоны ППП возникают в различных физических ситуациях, однако возможность образования поверхностного солитона СИП в двумерных анизотропных оптически поглощающих материалах ранее не изучалась.

В настоящей работе теоретически исследуется солитон СИП для ППП в фосфорене (или фосфореноподобных анизотропных двумерных материалах) для изучения влияния сильной анизотропной оптической проводимости фосфорена на солитон СИП.

Мы рассматриваем формирование оптического солитона СИП в фосфорене в случае, когда поперечно-магнитная ППП-мода с частотой $\omega \gg T^{-1}$ и шириной $T$ распространяется вдоль положительной оси $\xi$. Монослой (или очень тонкий слой) ЧФ и тонкий переходный слой толщиной $h$, который содержит небольшую концентрацию $n_{0}$ невзаимодействующих полупроводниковых квантовых точек (ПКТ) или оптических примесных атомов, зажаты между двумя полупространствами. Для простоты предположим, что оба полупространства линейны и изотропны, а $h \ll \lambda$, где $\lambda$ - длина волны ППП-моды. Среда 1 с диэлектрической проницаемостью $\varepsilon_{1}$ занимает область $z<0$, а среда 2 с диэлектрической проницаемостью $\varepsilon_{2}$ занимает область $z>h$. Мы рассмотрим четырехслойную структуру: среда $1 /$ фосфорен/резонансный

Письма в ЖТФ, 2018, том 44, вып. 8 


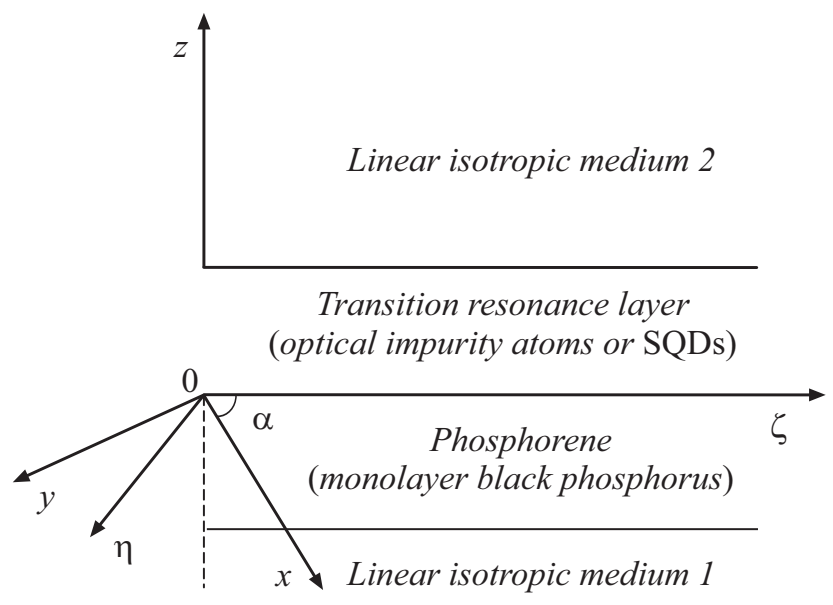

Направление распространения ППП-волны вдоль оси $\xi$. Оси координат $\xi, \eta$, $x$ и $y$ лежат в одной плоскости, перпендикулярной оси $z$. Направление оси $x$ (или направление „кресло“) и направление $y$ (или направление „зигзаг“) перпендикулярны оси $z$. Вектор электрического поля $\mathbf{E}$ поверхностной поперечномагнитной моды лежит в плоскости $\xi z$. Вектор магнитного поля волны Н параллелен оси $\eta$. Переходный резонансный слой толщиной $h$ содержит ПКТ или оптические примесные атомы. Фосфорен зажат между переходным слоем и средой 1. Вектор поляризации $\mathbf{P}$ примесных атомов или ПКТ направлен вдоль оси $\xi$.

переходный слой/среда 2 (см. рисунок). Анизотропия фосфорена в плоскости может быть выражена через декартовы координаты $(x, y, z)$ с тензором оптической проводимости

$$
\widehat{\sigma}=\left(\begin{array}{cc}
\sigma_{x x} & 0 \\
0 & \sigma_{y y}
\end{array}\right),
$$

где $\sigma_{x x}$ и $\sigma_{y y}$ - компоненты тензора проводимости вдоль направления „кресло“ (или направления вдоль оси $x$ ) и вдоль направления „зигзаг“ (или направления вдоль оси $y$ ) соответственно.

Для поперечно-магнитной ППП-моды электрическое поле $\mathbf{E}\left(E_{\xi}, 0, E_{z}\right)$ лежит в плоскости $\xi z$, перпендикулярной границе раздела между двумя полупространствами $(z=0)$, а магнитное поле $\mathbf{H}\left(0, H_{\eta}, 0\right)$

Письма в ЖТФ, 2018, том 44, вып. 8 
направлено вдоль оси $\eta$. Рассмотрим ситуацию, когда координатные оси $x, y, \xi$ и $\eta$ лежат в одной плоскости, перпендикулярной оси $z$. Функции

$$
\begin{aligned}
& U_{1}(\xi, z, t)=\int U_{1}(\Omega, Q) e^{\kappa_{1}(\Omega, Q) z+i(Q \xi-\Omega t)} d Q d \Omega, \quad z<0, \\
& U_{2}(\xi, z, t)=\int U_{2}(\Omega, Q) e^{-\kappa_{2}(\Omega, Q) z+i(Q \xi-\Omega t)} d Q d \Omega, \quad z>0
\end{aligned}
$$

являются разложением Фурье компонент электрического и магнитного полей $E_{\xi}, E_{z}, H_{\eta}$ поверхностной волны. Величины $\kappa_{1,2}^{2}=Q^{2}-\frac{\varepsilon_{1,2}}{c^{2}} \Omega^{2}$ характеризуют поперечную структуру ППП и определяются с помощью уравнений Максвелла в полупространствах 1 и 2.

Граничные условия для ППП при $z=0$ могут быть заданы формулами $[8-10]$

$$
H_{1, \eta}-H_{2, \eta}=\frac{4 \pi}{c}\left[\frac{\partial p}{\partial t}+\sigma(\alpha) E_{\xi}\right], \quad E_{1, \xi}=E_{2, \xi}, \quad D_{1, z}=D_{2, z} .
$$

Здесь $D_{1,2 ; z}-z$-компоненты вектора электрической индукции в полупространствах, $c$ - скорость света в вакууме. Поляризация резонансного переходного слоя дается формулой $\mathbf{P}(\xi, t)=\mathbf{e}_{\xi} p(\xi, t) \delta(z)$, где $\mathbf{e}_{\xi}-$ единичный вектор поляризации вдоль оси $\xi$. Плотность электрического тока монослоя фосфорена может быть представлена как $J_{\xi}=\sigma(\alpha) E_{\xi} \delta(z)$, где $\sigma(\alpha)=\sigma_{x x} \cos ^{2} \alpha+\sigma_{y y} \sin ^{2} \alpha-$ оптическая проводимость в произвольном направлении $\xi ; \alpha-$ угол между осями $\xi$ и $x$. Подставляя (1) в формулу (2), получим для $\xi$-компоненты электрического поля нелинейное волновое уравнение при $z=0$

$\int f(\Omega, Q) E_{\xi}(\Omega, Q) e^{i(Q \xi-\Omega t)} d Q d \Omega=-4 \pi p(\xi, t)-4 \pi \sigma(\alpha) \int E_{\xi}(\xi, t) d t$,

где

$$
f(\Omega, Q)=\sum_{l=1,2} \frac{\varepsilon_{l}}{\kappa_{l}} .
$$

В (3) учитывается, что $E_{\xi}(\Omega, Q)=E_{1 ; \xi}(\Omega, Q)=E_{2 ; \xi}(\Omega, Q)$.

Используя метод медленно меняющегося профиля волны $E_{\xi}=\sum_{l= \pm 1} \widehat{E}_{l} Z_{-l}$, поляризацию примесных атомов (ПКТ) можно 
записать как

$$
p(\xi, t)=-i n_{0} d \sum_{l= \pm 1} Z_{-l} \int g(\Delta) \rho^{l} d \Delta
$$

где $\widehat{E}_{l}$ и $\rho^{l}$ - медленно меняющиеся комплексные огибающие электрического поля и поляризации, $Z_{1}=\exp (i l(k \xi-\omega t))$ содержит быстро меняющуюся фазу несущей волны, $d-$ электрический дипольный момент атомов примесей или ПКТ, $\Delta=\omega_{0}-\omega, g(\Delta)-$ функция неоднородного уширения спектральной линии, $\omega_{0}$ - частота атомного (ПКТ) перехода. Величина $\rho^{l}$ определяется исходя из оптических уравнений Блоха [18]. Из волнового уравнения (3) получим закон дисперсии для ППП

$$
k^{2}=\frac{\omega^{2}}{c^{2}} \frac{\varepsilon_{1} \varepsilon_{2}}{\varepsilon_{1}+\varepsilon_{2}}
$$

и нелинейное уравнение для огибающей ППП при $z=0$

$$
\frac{\partial \widehat{E}_{-1}}{\partial \xi}+\frac{1}{V} \frac{\partial \widehat{E}_{-1}}{d t}=-\frac{4 \pi n_{0} d}{f_{Q}} \int g(\Delta) \rho^{-1} d \Delta-\frac{4 \pi \sigma(\alpha)}{\omega f_{Q}} \widehat{E}_{-1},
$$

где

$$
V=\frac{k c^{2}}{\omega} \frac{\varepsilon_{2} \tilde{\kappa}_{1}^{3}+\varepsilon_{1} \tilde{\kappa}_{2}^{3}}{\varepsilon_{2}^{2} \tilde{\kappa}_{1}^{3}+\varepsilon_{1}^{2} \tilde{\kappa}_{1}^{3}}, \quad \tilde{\kappa}_{1,2}^{2}=k^{2}-\frac{\varepsilon_{1,2}}{c^{2}} \omega^{2}, \quad f_{Q}=k\left(\frac{\varepsilon_{2}}{\tilde{\kappa}_{2}^{3}}+\frac{\varepsilon_{1}}{\tilde{\kappa}_{1}^{3}}\right) .
$$

Уравнение (4) для ППП с проводимостью является достаточно общим для описания различных нелинейных когерентных процессов образования нелинейных ППП в анизотропных двумерных материалах. Коэффициент затухания $\sigma(\alpha)$ в отличие от случая графена зависит от угла $\alpha$. Используя теорию возмущений для метода обратной задачи (ТМО3) [9,17], мы можем определить эволюцию параметров и профиль оптического солитона, когда анизотропная проводимость монослоя ЧФ $\sigma(\alpha) \neq 0$. Солитонное решение системы (4) для ППП в фосфорене имеет вид

$$
\widehat{E}_{-1}(\xi, \tau, \alpha)=\frac{2 \Lambda_{0} \hbar}{d} e^{i(\beta-2 \xi \tau)} \sec h\left[2 \Lambda\left(\tau-x_{0}\right)\right] e^{-\frac{4 \pi \sigma(\alpha)}{\omega f} \xi},
$$

где $\tau=t-\xi / V, 2 \Lambda_{0} \hbar / d-$ амплитуда импульса, $\omega-2 \xi-$ мгновенная частота, $\beta+2 \Lambda \xi / c-$ фаза при фиксированном значении $\xi, x_{0}-$

6 Письма в ЖТФ, 2018, том 44, вып. 8 
центральное положение импульса, которое определяет время задержки солитона в среде, $\Lambda_{0}-$ начальное значение $\Lambda$ при $\xi=0, \hbar-$ постоянная Планка.

Формулы (1), (5) и (6) определяют параметры поверхностного солитона при любых значениях $z, \xi$ и $t$, которые зависят от оптических атомов (ПКТ) через величину $d$, а также от диэлектрических проницаемостей двух соприкасающихся сред $\varepsilon_{1,2}$, поперечной структуры ПППмоды $\kappa_{1,2}$, проводимости фосфорена $\sigma$ и направления распространения через угол $\alpha$. Из (6) видно, что зависящая от $\alpha$ оптическая проводимость фосфорена $\sigma(\alpha)$ экспоненциально уменьшает амплитуду поверхностного солитона СИП в процессе распространения. Направления распространения, соответствующие максимальному и минимальному затуханию амплитуды, лежат вдоль направлений оси $x$ („кресло“) и оси $y$ („зигзаг“) нанопленки ЧФ соответственно. Наиболее быстрое затухание интенсивности происходит, когда импульс распространяется вдоль направления „кресло“, $\alpha=0$. Следует отметить, что представленный подход (ТМО3) является довольно общим и наряду с фосфореном может также применяться для других двумерных анизотропных материалов (например, для различных типов двумерных полупроводников: $h$ $\mathrm{BN}, \mathrm{MoSe}_{2}, \mathrm{WSe}_{2}$ и т.д. $\left.[18,19]\right)$, а также в случае, когда проводимость среды $\sigma$ зависит не только от угла $\alpha$, но и от волнового числа $k$ и частоты $\omega$ несущей волны $[1,20]$. Более того, здесь мы рассмотрели случай аттенюатора, но этот подход может также применяться для усиливающей среды, экспериментально исследованной в [12-16]. Параметры оптического солитона в анизотропных и изотропных поглощающих средах имеют один и тот же порядок, и, следовательно, мы надеемся, что это явление будет наблюдаться экспериментально, а анизотропные оптические свойства поверхностного солитона в монослое (а также в тонком слое) ЧФ могут стимулировать развитие устройств, работающих на основе фосфорена.

Работа выполнена в рамках проекта № 217064 ННФ им. Ш Руставели.

\section{Список литературы}

[1] Carvalho A., Wang M., Zhu X., Rodin A.S., Su H., Neto A.H.C. // Nature Rev. Mater. 2016. V. 1. P. 16061.

[2] Bao Z.-W., Wu H.-W., Zhou Y. // Appl. Phys. Lett. 2016. V. 109. P. 241902.

Письма в ЖТФ, 2018, том 44, вып. 8 
[3] Geim A.K., Novoselov K.S. // Nature Mater. 2007. V. 6. P. 183-191.

[4] Liu H., Neal A.T., Zhu Z., Xu X., Tomanek D., Ye P.D. // ACS Nano. 2014. V. 8. P. 4033-4041.

[5] Zhang S., Wang N., Liu S., Huang S., Zhou W., Cai B., Xie M., Yang Q., Chen X., Zeng H. // Nanotechnology. 2016. V. 27. P. 274001.

[6] Kamal C., Chakrabarti A., Ezawa M. // Phys. Rev. B. 2016. V. 93. P. 125428.

[7] Adamashvili G.T. // Physica B. 2014. V. 454. P. 45-49.

[8] Адамашвили Г.Т. // Оптика и спектроскопия. 2015. Т. 119. № 2. С. 265-270.

[9] Adamashvili G.T., Kaup D.J. // Phys. Phys. A. 2017. V. 95. P. 053801.

[10] Адамашвили Г.Т., Адамашвили Н.Т., Пейкришвили М.Д., Моцонелидзе Г.Н., Коплатадзе Р.Р. // Письма в ЖТФ. 2009. Т. 35. В. 1. С. 35-40.

[11] Nesterov M.L., Bravo-Abad J., Nikitin A., Garcia-Vidaland F., MartinMoreno L. // Laser Photon. Rev. 2013. V. 7. P. L7-L11.

[12] Sotor J., Sobon G., Macherzynski W., Paletko P., Abramski K.M. // Appl. Phys. Lett. 2015. V. 107. P. 051108.

[13] Li D., Jussila H., Karvonen L., Ye G., Lipsanen H., Chen X., Sun Z. // Sci. Rep. 2015. V. 5. P. 15899.

[14] Wang Z., Xu Y., Dhanabalan S.C., Sophia J., Zhao C., Xu C., Xie Y., Xiang Q., Li J., Zhang H. // IEEE Photon. J. 2016. V. 7. P. 1503310.

[15] Song Y., Chen S., Zhang Q., Li L., Zhao L., Zhang H., Tang D. // Opt. Express. 2016. V. 24. P. 25933-25942.

[16] Du J., Zhang M., Guo Z., Chen J., Zhu H., Hu G., Peng P., Zheng Z., Zhang H. // Sci. Rep. 2017. V. 7. P. 42357.

[17] Adamashvili G.T., Kaup D.J., Knorr A. // Phys. Rev. A. 2014. V. 90. P. 053835.

[18] Cassabois G., Valvin P., Gil B. // Nature Photon. 2016. V. 10. P. 262-266.

[19] Castellanos-Gomez A. // Nature Photon. 2016. V. 10. P. 202-204.

[20] Saberi-Pouya S., Vazifehshenas T., Salavati-Fard T., Farmanbar M., Peeters F.M. // arXiv:1704.05266v1 (condmat.mes-hall) (18 Apr. 2017).

$6^{*}$ Письма в ЖТФ, 2018, том 44, вып. 8 